\title{
Periodization in Anterior Cruciate Ligament Rehabilitation: A Novel Framework
}

\author{
George Kakavas $^{a} \quad$ Nikolaos Malliaropoulos $^{b, c, d} \quad$ Georgios Bikos $^{\mathrm{e}}$ Ricard Pruna \\ Xavier Valle ${ }^{f}$ Panagiotis Tsaklis ${ }^{g, h}$ Nicola Maffulli, ${ }^{i, j, k}$ \\ ${ }^{a}$ Fysiotek Spine and Sports Lab Athens, Athens, Greece; b Sports and Exercise Medicine Clinic, Asklipiou, \\ Thessaloniki, Greece; ' Sports Clinic, Rheumatology Department, Barts Health NHS Trust, London, UK; ${ }^{d}$ Centre for

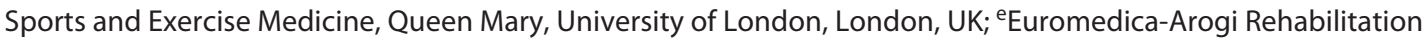 \\ Clinic, Pylaia, Thessaloniki, Thessaloniki, Greece; ${ }^{\prime}$ Medical Services Ciutat Esportiva Barcelona, FC Barcelona, \\ Barcelona, Spain; ${ }^{9}$ Biomechanics and Ergonomics, ErgoMech Lab, Department of Physical Education and Sport \\ Science, University of Thessaly, Trikala, Greece; ${ }^{\text {h}}$ Department of Molecular Medicine and Surgery Growth and \\ Metabolism, Karolinska Institutet, Stockholm, Sweden; 'London Sports Care, BMI London Independent Hospital, \\ London, UK; 'Department of Orthopaedics, School of Medicine, Surgery and Dentistry, Salerno, Italy; ${ }^{\mathrm{k} S c h o o l}$ of \\ Pharmacy and Bioengineering, Keele University School of Medicine, Stoke on Trent, Stoke on Trent, UK
}

\section{Highlights of the Study}

- Periodization should be considered not only for athletic performance but also for rehabilitation of anterior cruciate ligament injury.

- Anterior cruciate ligament injury is not just a local mechanical trauma, but its effects extend to the central nervous system as well.

- Rehabilitation planned according to the periodization concepts should allow better integration of the needs of the patients to return to sport, using concepts which will be easily integrated with the sports and strength and conditioning coaches to ultimately benefit the athlete and prevent reinjury.

\section{Keywords}

Periodization - Anterior cruciate ligament · Rehabilitation · Brain · Neurocognitive function · Neuroplasticity

\section{Abstract \\ More than 250,000 anterior cruciate ligament (ACL) injuries occur each year in the USA, and approximately $65 \%$ of these injuries undergo reconstructive surgery. Appropriate reha- bilitation after ACL reconstruction can yield predictably good outcomes, with return to previous levels of activity and}

high knee function. At present, periodization is used at all levels of sports training. Whether conceptualized and directed by coaches, or by athletes themselves, competitors structure their training in a cyclic fashion, enabling athletes to best realize their performance goals. In practical application, sport physical therapists use periodization: postoperative "protocols" serve as rudimentary forms of periodization, albeit implemented over shorter time frames than that typically employed in preparation for competition. An ACL injury should not be considered a "simple" musculoskeletal pathology with only local mechanical or motor dysfunctions.
(C) 2020 The Author(s)

Published by S. Karger AG, Basel

This is an Open Access article licensed under the Creative Commons Attribution-NonCommercial-4.0 International License (CC BY-NC) (http://www.karger.com/Services/OpenAccessLicense), applicable to the online version of the article only. Usage and distribution for commercial purposes requires written permission.
Nicola Maffulli

Department of Trauma and Orthopaedics, Faculty of Medicine Surgery and Dentistry, University of Salerno, Baronissi Campus Via S Allende, Baronissi, Salerno (Italy) n.maffulli@qmul.ac.uk 
Together with the psychological trauma and reduction in physical capacity, there is a cascade of events, including neurological insult to the central nervous system and reduction in afferences to the sensorimotor system. Rehabilitation should consider all these issues, and periodization would allow to better define and to plan aims and objectives to return athletes to their sport. Technological resources including advanced neuroimaging methods, virtual reality for injury risk screening and return to sport assessment, and interactive artificial reality-based neuromuscular training methods offer new approaches and tools to address this important biomedical problem. The cost and availability of many of these technologies will continue to decrease, providing greater availability, scientific rigor, and ultimately, utility for cost-effective and data-driven assessments.

(C) 2020 The Author(s)

Published by S. Karger AG, Basel

\section{Introduction}

More than 250,000 anterior cruciate ligament (ACL) injuries occur each year in the USA, and approximately $65 \%$ of these injuries are treated with reconstructive surgery [1]. A consistent approach to rehabilitation after ACL reconstruction can yield good outcomes, with predictably return to previous levels of activity [2]. In 1996, researchers at the University of Delaware published criterion-based rehabilitation guidelines, in which progression was established according to specific clinical milestones following tissue-healing time frames [3]. Criterion-based guidelines aim to maximize patients' response to exercise at the current level of function, while minimizing the risk of injury to the healing tissue. Based on sound principles and current evidence, those guidelines have continued to guide rehabilitative care. Rehabilitation after ACL reconstruction has continued to move away from surgery-led rehabilitation, in which surgery constrains the progression of the rehabilitation process, and toward rehabilitation-modified surgery, in which the reconstruction techniques are robust enough to withstand early mobilization, conditioning, and strengthening exercises [4].

Periodization, that is, dividing of the annual training plan into smaller distinct phases to introduce more manageable segments, is a sophisticated method of preparation for competition. The foundations of modern periodization in sport were developed in the Soviet Union around 1 century ago. Kotov, in 1917, divided training into general, preparatory, and specific stages, and Ozolin, in 1949, stressed the importance of considering the competitive calendar and climatic factors in periodization [5].
At present, periodization is used at all levels of sports training. Whether conceptualized and directed by coaches or by the athletes themselves, competitors at all levels of performance structure their training in a cyclic fashion to optimize their performance capacities and goals [4].

Matveyev summarized the modern concept of periodization by dividing the training year into distinct phases, each with different characteristics and special application to train endurance or strength and power athletes [6]. The fundamental concepts presented by Matveyev were applied to training in many sports, including track and field, swimming, rowing, cycling, skiing, weightlifting, or skating [3]. The increasing number of competitions together with the improvement in technology and facilities led to further variation of periodization structures with the introduction of double or triple peaked periodized cycles [7].

In most sports, the annual training calendar is conventionally divided into 3 cyclic phases of training: the preparatory phase, the competitive phase, and the transition phase [8]. The preparatory and competitive phases are further divided into subphases, since the goals of training, and subsequently the specificity of training tasks, can vary substantially at different points in the annual training plan [9]. The preparatory phase has both general and specific subphases based on the different characteristics of training, and the competitive phase usually follows a short precompetitive subphase. The transition phase, also commonly known as active rest, allows athletes' mind and body to recuperate from the rigors of the competitive phase and to get ready for the preparatory phase to follow [10]. Additionally, each phase is composed of smaller periods, known as macrocycles and microcycles. The specific objectives of these smaller segments derive from the general objectives of the annual training plan. Building upon these concepts, the integration of training principles described in the section on foundation training concepts depicts the relationships existing between training intensity and duration throughout the training year [11].

Most healthcare and fitness professionals typically think of exercise as a universally positive activity, which has beneficial effects on the human body. However, this viewpoint is only partially correct. In relation to the effects on the human body, any physical activity can be interpreted as a physiological stressor [12]. Thus, when physical activity is properly dosed in individuals who are physiologically capable to acutely respond to the stress imposed, homeostasis is restored. With repeated bouts of properly dosed physical activity in individuals who are physiologically capable to respond over longer time 
frames, adaptation occurs. Conversely, when the body tissues and systems are overly disrupted, disease and injuries occur, as an individual cannot respond acutely or over longer time frames.

Decades ago, sport scientists started to take note of Selye's general adaptation syndrome (GAS), whereby systems will adapt to changes which they might experience in an attempt to meet the demands of the stressors, and began applying its potential benefits to athletic training and competition. According to GAS theory, the fatigue induced by physical activity is temporary, and reversible if the training load is appropriate. Accordingly, factors such as proper exercise technique, rest, and nutrition are keys to ensuring that athletes can recover from the stress of any given training bout [13].

Periodization can be traced to Selye's GAS. A periodized program aims to optimize the principle of overload, the process by which the neuromuscular system adapts to unaccustomed loads or stressors [12]. The training program specifies the intensity, volume, and frequency; the interactions of these variables result in the overload. For the neuromuscular system to maximally adapt to the training load or stress, volume and intensity alterations are necessary. Given the increased demands, the neuromuscular system adapts with increases in muscular performance. If the system is allowed to adapt to stressors without concomitant changes in overload, no further adaptations are needed, and increases in the desired outcome will eventually stop. Conceptually, periodization helps avoid this problem because the load on the neuromuscular system is constantly changing. Furthermore, periodization may be beneficial by adding variation to workouts, thus avoiding boredom or training plateaus [14].

Although other models of periodization exist, there are 2 primary models. First, the classic, or linear, model is based on changing exercise volume and load across several predictable mesocycles [15]. Based on a 12-month period, the program is referred to as a macrocycle; the 2 subdivisions are the mesocycle (3-4 months) and the microcycle (1-4 weeks). The other main model is the undulating periodization model [15]. The term nonlinear periodization has become more favorable compared to undulating periodization. Nonlinear periodization is based on the concept that volume and load are altered more frequently (daily, weekly, and biweekly) to allow the neuromuscular system more frequent periods of recovery. Phases are much shorter, providing more frequent changes in stimuli, which may be highly conducive to, for example, strength gains. Kraemer et al. [16] expanded this concept by including planned versus flexible nonlinear periodization. The planned model follows predicted loading schemes, but the flexible plan allows adjusting the plan based on the status of the athlete. Last, reverse linear periodization follows the modifications in load and volume but in the reverse order: increasing volume and decreasing load [16].

Periodization can be accomplished by manipulating sets, repetitions, exercise order, number of exercises, resistance, rest periods, type of muscle contraction, and training frequency, thereby providing numerous periodization programs. Manipulating variables is arguably the greatest challenge that coaches face, for example, when designing and modifying resistance training programs [17].

Thus, it is necessary to point out important differences in the ways these variables are typically conceptualized in rehabilitation and in periodization models. One major difference between such models is the typical time frames of rehabilitation and periodization models. While some patients are certainly seen and followed for longer periods, most patients participate in physical therapy rehabilitation programs between 6 and 12 weeks [18]. Physical therapists manipulate the intensity, duration, frequency, mode volume, and specificity of activity completed in each rehabilitation session, with the goal of stimulating increased function for the patient in question [18]. These individuals are then typically discharged from care, and in most instances are not seen again by the treating physical therapist. On the contrary, coaches and/or athletes themselves manipulate these same variables over much longer time frames, such as the whole year.

In practical application, sport physical therapists use periodization, a common example being postoperative "protocols" that serve as rudimentary forms of periodization, albeit implemented over shorter time frames than that typically used in preparation for competition. An example would be the postoperative progression of an athlete recovering from ACL reconstruction. This brief periodization plan initially focuses on the preparatory aspects of rehabilitation, addressing impairments such as range of motion, quadriceps strength, and impaired gait. These attributes of functional limitation are typically addressed within the first 6-8 weeks following surgery. Once addressed, there is a transition phase where the focus turns to more function-based recovery; as an athlete reaches this transition mesocycle, we can further divide it into 4 microcycles: dynamic stabilization and core strengthening, functional strength, power development, and sport performance symmetry [19]. 
This brief periodization plan includes entrance criteria, regular testing and measures for advancement, and modifications of exercise prescription. This scenario requires time to complete a microcycle fully before progression to the next microcycle. Ideally, this would be the case not only for the athlete's safety and prevention of reinjury, but also for the potential to return to sports at higher-than-previous levels. By fully understanding the goals of the athlete and the time when the injury occurred during the training calendar, one can best help the athlete return to the proper periodization subphase. This means that postoperative rehabilitation following ACL reconstruction must consider more than the strength ratios of the involved and uninvolved legs but also the state of the athletes' aerobic and anaerobic capacities, neurological conditioning, and physiological readiness before clearing them for full return to competition [20].

\section{Application of Periodization to Rehabilitation}

Rehabilitation programs have traditionally used a basic progressive overload approach primarily focusing on the injured area. Periodized training is a safe method of training for older adults as well as those in pain [11]. The ideas and concepts presented in the present article have not been tested in randomized controlled trials, and they may stimulate further suitable studies. For example, specific guidelines on resistance training variables are consistently lacking in rehabilitation protocols. The clinician has general goals for each phase and precautions and a vague incomplete list of exercises to be performed. Studies have compared eccentric training to a standard rehabilitation protocol after an ACL reconstruction, open versus closed chain exercises in ACL-deficient knee rehabilitation and patellofemoral pain, and even home versus physical therapy-supervised rehabilitation based on a standard rehabilitation protocol $[2,6,13]$.

In ACL reconstruction, several options exist. In addition to comparing linear versus nonlinear models using standard rehabilitation protocols, researchers could compare modes or duration of training. Using linear and nonlinear periodization as a framework, researchers could allocate patients to be rehabilitated following an ACL reconstruction into 1 of the 3 groups: control, linear, and nonlinear. Traditional rehabilitation programs could be compared to eccentrically based programs, or eccentrics could be used in the periodization models. In post-surgical ACL reconstruction patients, endurance, hypertrophy, strength, and power days can be developed, using a 3-day-per-week rehabilitation program. When an athlete progresses to more sport-specific drills (plyometrics and agility) in the later stages of rehabilitation, emphasis can shift to strength, power, and hypertrophy sessions [21]. In the final stages of rehabilitation before return to sport, power, strength, or hypertrophy can be emphasized based on the athlete's deficits. If an athlete has persistent quadriceps deficits, hypertrophy sessions may be beneficial. Once the patient reaches the fourth postoperative week after the ACL reconstruction, emphasis can be shifted to muscle endurance [9]. This provides the baseline for hypertrophy and strength; power training will eventually be implemented. The hypertrophy phase should be introduced around the eighth postoperative week because initial muscular gains arise from neuromuscular efficiency mechanisms as opposed to muscular hypertrophy. Strength training should be the emphasis between the 12 th and 16th weeks before power-type training. In the power phase of a program, 2 sessions per week are sufficient while still providing adequate recovery between sessions [22].

Nonlinear programs still place emphasis on a given parameter (endurance, strength, power, etc.) while allowing for increased flexibility on how to pursue this goal [9]. Different training phases in many linear plans have a specific training goal, such as hypertrophy, strength, strength/ power, and power. One goal of many linear periodization programs is to maximize strength/power after the last training phase, typically termed a power phase. With nonlinear periodization, training intensity and volume are changed much more frequently [23]. The early weeks primarily emphasize endurance, but hypertrophy and strength sessions are introduced. In week 8-12, the emphasis is on muscle hypertrophy, similar to the linear program. The overall goals are also similar in both programs. In summary, the concept of periodization in terms of strength training has shown promise in strength and conditioning on healthy trained and untrained athletes [24]. There is a paucity of data in rehabilitation research using the principles of periodization in the design of rehabilitation programs [7].

\section{Current Rehab Stages for ACL Rehabilitation}

In isolated ACL reconstruction, protocols were developed around evidence-based medicine, with consideration given to biological healing time frames. As a result, the treatment of an isolated ACL reconstruction has required only minor adjustments to reflect recent advances 
in the rehabilitation literature and surgical techniques. Time frames have been included to guide the therapist, and patients are required to meet the various clinical milestones prior to advancing to the next stage, regardless of the time frame. Clarification and adjustments of clinical milestones and additions of interventions aim to improve functional outcomes and to provide a safe return to sports [6].

In general, the following 5 phases are recommended when dealing with these patients: an immediate postoperative phase followed by early and intermediate postoperative phases are the 3 stages with marked neurocognitive deficits, accounting for the low mobility tasks in those phases. The late postoperative and transitional phases contain more tasks such as running and jumping and cutting drills, but we do not have accurate and valid tests to ascertain when proprioception from the graft returns and motor control is well established [12]. Indeed, it is possible that neuromuscular adaptations are more important than anatomical/histological evidence of maturation of the graft.

An ACL injury induces changes to the central nervous system (CNS), given the loss of information from mechanoreceptors, pain and developed motor compensations. This neuroplastic disruption progresses until altered motor strategies potentially become the norm. Subsequent restoration of baseline function then becomes a must against maladaptive neuroplasticity developed in the wake of altered CNS input and motor output compensations.

A most interesting and widely unexplored aspect of implicit learning in rehabilitation is its connection with anticipation and decision-making [8]. This may be important in the late stages of rehabilitation, when athletes are approaching the return to sport phase. An athlete should be progressively exposed to physical, environmental, and psychological stressors comparable to those to which they will be exposed to in their actual sport. Considering secondary ACL injury prevention, training in this phase of the rehabilitation process should emphasize motor control factors such as anticipation, responses to perturbation, and visual-motor control within complex task environmental interactions [25].

Neuroplasticity deficits following ACL injury may at least in part be caused by physiotherapy that does not engage differential learning. Over the course of rehabilitation following injury, excitability of the motor cortex for quadriceps contractions decreased [26], at least partially from the lack of differential exercise approaches that do not force the motor cortex to reintegrate the memory trace for quadriceps motor control before each repetition. Neurophysiological data across the stages of rehabilita-

Periodization in Anterior Cruciate

Ligament Rehabilitation tion are lacking. Lepley et al. [26] tried to quantify neuroplasticity longitudinally after an injury, and the ability of differentially learning to modify sensorimotor neural processing may provide a means for therapists to target neural activity in a way that standard therapy does not [27].

Rehabilitation programs mainly focus on pre-planned motor skills in a predictable environment with a focus on postural alignment. Practicing these narrow motor skills fails to comprehensively address the interaction between sensory cues and motor responses as they relate to specific sports activities of an athlete in task and environmental constraints on the field. Following ACL reconstruction, athletes should progressively face the physical, environmental, and psychological stressors to which they will be exposed, in their sport, as part of a comprehensive and progressive return to sport continuum [24].

Following an ACL tear, the CNS may increase its reliance on alternative sensory sources, such as visual-feedback and spatial awareness [14]. Neuroimaging has been used to quantify brain activation differences between subjects with ACL deficiency who did not return to previous levels of physical activity and a healthy control group. ACL-deficient individuals exhibited increased activation in the posterior inferior temporal gyrus (visual processing), pre-supplementary motor area (motor planning), and secondary somatosensory area (pain and sensory processing) [28].

The depressed excitability of the motor cortex suggests that greater motor cortex activation is required to achieve motor drive and/or that motor cortex input from the rest of the brain in the form of structural or functional connectivity must increase to achieve adequate motor drive. The ACL may tear in $<70 \mathrm{~ms}$, but the earliest reflexive activity for dynamic restraint requires at least $35 \mathrm{~ms}$ to begin developing muscle tension [29]. Additionally, cognitive appreciation of any coordination errors can take up to $500 \mathrm{~ms}$. Therefore, the high angular velocities and forces associated with sports participation require advanced cognitive planning through feed-forward motor control. Otherwise, overreliance on reflexive strategies for dynamic stability may be insufficient to protect the (reconstructed) ACL [6].

Increased physiological knee valgus, load-reduced neurocognitive function, increased joint laxity, small femoral notch width, and altered neuromuscular properties have been considered as potential risk factors specific to young females [30]. All these factors are potential interventions to target the relevant processes. A further ACL injury following successful reconstruction has been 
reported to up to $23 \%$ in athletes younger than 25 years when returning early to competitive sports involving jumping and cutting activities [18], and a major aim of rehabilitation is to prevent a reinjury.

Restoration of symmetry alone is not sufficient to reduce the risk of reinjury. Focus should also be placed on addressing underlying deficits which likely contributed to the primary ACL injury. In addition, a series of inciting events are likely to occur prior to the actual injury, and different playing situations provide further complexity [11]. For example, ball possession, position of team mates, and actions of opponents all impose different challenges and problems for athletes to solve. Thus, perceptual capacities play an important role in team and ball sports by enhancing perception in rapidly changing environments. Interpreting situational information correctly and efficiently allows them to select the most appropriate response. The impact of this complex environment should not be ignored when second ACL injury prevention is the goal. ACL injuries during sport are predominantly noncontact, suggesting that an injury may be the product of sensorimotor errors that result in a neuromuscular control strategy unable to accommodate deleterious joint loading [31]. Furthermore, the vast majority of noncontact injuries occur while athletes are cognitively distracted, attending to complex visual demands or environmental stimuli [10], suggesting that neural mechanisms may directly contribute to the athlete's ability to safely interact with the dynamic sport environment [32].

The term sensory retraining describes how the CNS integrates separate sensory stimuli (e.g., visual, vestibular, and proprioceptive) by weighting them according to reliability, essentially decreasing the weight of unreliable stimuli and increasing the weight of others. Injury reduction and rehabilitation should include a much broader array of drills and practice scenarios with increasing levels of cognitive complexity to ensure adequate exposure and heightened readiness to return to optimal performance [28]. In addition, involving players and coaches in the design and/or selection of sport-specific exercises may prove fruitful by increasing the perception of ownership and adherence. Perceptual and cognitive loads must be viewed in the same light as the physical components of performance that we devote so much of our time towards [33]. Traditional musculoskeletal rehabilitation approaches tend not to integrate motor learning principles explicitly or with a goal to induce neuroplasticity, or sensory reweighting, or virtual reality technologies that support optimized functional performance and recovery. Incorporation of these new technologies and therapies (strobe goggles, multiple object tracking [MOT], etc.) may provide a means to reduce the high reinjury rate after ACL reconstruction, as the noncontact ACL injury event is essentially a coordination error in sensory, visual, or motor processing [34]. Furthermore, emerging evidence has demonstrated the existence of CNS changes following acute traumatic knee injuries, which may influence motor control and functional outcomes of ACLR patients. As such, motor learning strategies, and other modalities, may constitute a potential solution to mitigate neuroplastic effects of injury that can impede rehabilitative progress [35].

\section{Practical Application}

Traditionally, laboratory-based biomechanical assessment of sport-relevant tasks, such as landing, jumping, and cutting, have been used to assess neuromotor deficits that are purported to increase athletes' risk of sustaining musculoskeletal injury during sport $[36,37]$. These assessments typically involve athletes performing a battery of tests in a standard, systematic manner (i.e., according to a prescribed set of instructions and in a specific order) and are subsequently used as proxies for assessing how these athletes are likely to perform in real-world sport environments [38]. The constrained nature of task performance in a laboratory setting differs significantly from the dynamic, sport-specific contexts in which athletes incur injury [39]. This may partially explain why interventions based on traditional biomechanical assessments are often ineffective at reducing injury and especially reinjury rates. Although on-field, sport-specific assessments would provide a solution to this issue, several factors make this impractical [40].

Technological resources including advanced neuroimaging methods, virtual reality for injury risk screening and return to sport assessment, and interactive artificial reality-based neuromuscular training methods offer new approaches and tools for researchers and clinicians to address this biomedical issue. The cost and availability of many of these technologies will continue to decrease, providing greater availability, scientific rigor, and ultimately, utility for cost-effective and data-driven assessments.

\section{Conclusion}

An ACL injury should not be considered a "simple" musculoskeletal pathology with only local mechanical or motor dysfunctions. Together with the psychological 
trauma and reductions in physical capacity, there is a cascade of likely events across the whole spectrum, including neurological insult to the CNS and reduction in afferences to the sensorimotor system. Injuries of any type hinder the capacity of athletes to reach their training or competition goals and often necessitate the intervention of a sport physical therapist. Sport rehabilitation specialists should have a basic understanding of periodization theory. Such an understanding can help sport medicine teams to better interact with the competitive mindset of athletes, their coaches, and their goals. A basic understanding of these theories and models may help sport rehabilitation specialists to skillfully plan rehabilitation programs that then progress toward the realization of the patients' treatment goals. Such an understanding allows well-informed sport rehabilitation specialists to better bridge the gap between training to prepare for competition widely used by coaches and the treatment of injuries that may occur along the way. A strong link has been demonstrated between acquisition of motor skills and neuronal plasticity at cortical and subcortical levels; this link evolves over time and engages different spatially distributed interconnected brain regions. We advocate a neurocognitive approach when incorporating these principles and modalities, which should not replace exercise or rehabilitative goal, and should be used as adjuncts during the exercises already prescribed. The fundamentals of rehabilitation, including range of motion, strength recovery, and basic movement pattern restoration, should still remain primary goals of rehabilitation.

\section{Statement of Ethics}

The authors have no ethical conflicts to disclose.

\section{Conflict of Interest Statement}

The authors have no conflicts of interest to declare.

\section{Funding Sources}

This study received no specific grant from any funding agency in the public, commercial, or not-for-profit sectors.

\section{Author Contributions}

George Kakavas planned the study and contributed to write the manuscript. Nikolaos Malliaropoulos, Georgios Bikos, Ricard Pruna, Xavier Valle, and Panagiotis Tsaklis contributed to write the manuscript. Nicola Maffulli contributed, wrote, and edited the manuscript. All the authors reviewed and revised the manuscript and approved the final version.

\section{References}

1 Baker D. Applying the in-season periodization of strength and power training to football. Strength Cond J. 1998;20(2):18-27.

2 Naclerio F, Moody J, Chapman M. Applied periodization: a methodological approach. J Hum Sport Exercise. 2013;8:350-66.

3 Siff MC. Supertraining. Denver, CO: Supertraining Institute; 2004. p. 83-5.

4 Bompa TO, Haff G. Periodization: theory and methodology of training. 5th ed. Leeds: Human Kinetics; 2009.

5 Graham J. Periodization research and an example application. Strength Cond J. 2002;24: $62-70$.

6 Lorenz DS, Reiman MP, Walker JC. Periodization: current review and suggested implementation for athletic rehabilitation. Sports Health. 2010;2(6):509-18.

7 Issurin VB. New horizons for the methodology and physiology of training periodization: block periodization: new horizon or a false dawn? Sports Med. 2010;40(9):803-7.

8 Plisk SS, Stone MH. Periodization strategies. Strength Cond J. 2003;25(6):19-37.

9 Harries SK, Lubans DR, Callister R. Systematic review and meta-analysis of linear and undulating periodized resistance training programs on muscular strength. J Strength Cond Res. 2015;29(4):1113-25.

10 Haff GG. Periodization of training. In: Chandler TJ, Brown LE, editors. Conditioning for strength and human performance. Lippincott Williams and Wilkins; 2012. p. 326-43.

11 Hoover DL, VanWye WR, Judge LW. Periodization and physical therapy: bridging the gap between training and rehabilitation. Phys Ther Sport. 2016;18:1-20.

12 Selye H. Stress without distress. Philadelphia, PA: Lippincott; 1974. p. 1-171.

13 Gamble P. Periodization of training for team sports athletes. Strength Cond J. 2006;28(5): 56-66.

14 Davies GJ, McCarty E, Provencher M, Manske RC. ACL return to sport guidelines and criteria. Curr Rev Musculoskelet Med. 2017; 10(3):307-14.

15 Poliquin C. Football: five steps to increasing the effectiveness of your strength training program. National Strength Cond Assoc J. 1988;10(3):34.

16 Kraemer WJ, Fleck SJ, Evans WJ. Strength and power training: physiological mecha- nisms of adaptation. Exerc Sport Sci Rev. 1996;24:363-97.

17 Sugimoto D, LeBlanc JC, Wooley SE, Micheli LJ, Kramer DE. The effectiveness of a functional knee brace on joint-position sense in anterior cruciate ligament-reconstructed individuals. J Sport Rehabil. 2016;25(2):190-4.

18 Boggenpoel BY, Nel S, Hanekom S. The use of periodized exercise prescription in rehabilitation: a systematic scoping review of literature. Clin Rehabil. 2018;32(9):1235-48.

19 Di Stasi S, Myer GD, Hewett TE. Neuromuscular training to target deficits associated with second anterior cruciate ligament injury. J Orthop Sports Phys Ther. 2013;43(11):777.

20 Relph N, Herrington L. The effect of conservatively treated $\mathrm{ACl}$ injury on knee joint position sense. Int J Sports Phys Ther. 2016;11: 536-43.

21 Gabbett T. Infographic: the training-injury prevention paradox: should athletes be training smarter and harder? Br J Sports Med. 2018;52(3):203.

22 Boden BP, Dean GS, Feagin JA Jr, Garrett WE Jr. Mechanisms of anterior cruciate ligament injury. Orthopedics. 2000;23(6):573-8.
Periodization in Anterior Cruciate

Ligament Rehabilitation
Med Princ Pract 2021;30:101-108

DOI: $10.1159 / 000511228$ 
23 Miranda F, Simão R, Rhea M, Bunker D, Prestes J, Leite RD, et al. Effects of linear vs. daily undulatory periodized resistance training on maximal and submaximal strength gains. J Strength Cond Res. 2011;25(7):1824-30.

24 Adams D, Logerstedt DS, Hunter-Giordano A, Axe MJ, Snyder-Mackler L. Current concepts for anterior cruciate ligament reconstruction: a criterion-based rehabilitation progression. J Orthop Sports Phys Ther. 2012; 42(7):601-14

25 Wilk KE, Arrigo CA. Rehabilitation principles of the anterior cruciate ligament reconstructed knee: twelve steps for successful progression and return to play. Clin Sports Med. 2017;36(1):189-232.

26 Lepley LK, Wojtys EM, Palmieri-Smith RM. Combination of eccentric exercise and neuromuscular electrical stimulation to improve quadriceps function post-ACL reconstruction. Knee. 2015;22(3):270-7.

27 Greenberg EM, Greenberg ET, Albaugh J, Storey E, Ganley TJ. Rehabilitation practice patterns following anterior cruciate ligament reconstruction: a survey of physical therapists. J Orthop Sports Phys Ther. 2018;48: 801-11.
28 Grooms DR, Myer GD. Upgraded hardware horizontal line what about the software? Brain updates for return to play following ACL reconstruction. Br J Sports Med. 2017; 51:418-9.

29 Kaeding CC, Léger-St-Jean B, Magnussen RA. Epidemiology and diagnosis of anterior cruciate ligament injuries. Clin Sports Med. 2017;36(1):1-8.

30 Reiman MP, Lorenz DS. Integration of strength and conditioning principles into a rehabilitation program. Int $\mathrm{J}$ Sports Phys Ther. 2011;6(3):241-53.

31 Grooms D, Appelbaum G, Onate J. Neuroplasticity following anterior cruciate ligament injury: a framework for visual-motor training approaches in rehabilitation. J Orthop Sports Phys Ther. 2015;45(5):381-93.

32 Montalvo AM, Schneider DK, Silva PL, Yut L, Webster KE, Riley MA, et al. "What's my risk of sustaining an ACL injury while playing sports?" A systematic review with meta-analysis. Br J Sports Med. 2019;53:1003-12.

33 Mejane J, Faubert J, Romeas T, Labbe DR. The combined impact of a perceptual-cognitive task and neuromuscular fatigue on knee biomechanics during landing. Knee. 2019;26(1): 52-60.

34 Faubert J, Sidebottom L. Perceptual-cognitive training of athletes. J Clin Sport Psychol. 2012;6(1):85-102.
35 Appelbaum LG, Schroeder JE, Cain MS, Mitroff SR. Improved visual cognition through stroboscopic training. Front Psychol. 2011;2: 276

36 Koga H, Nakamae A, Shima Y, Iwasa J, Myklebust G, Engebretsen L, et al. Mechanisms for noncontact anterior cruciate ligament injuries: knee joint kinematics in 10 injury situations from female team handball and basketball. Am J Sports Med. 2010;38: 2218-25.

37 Kakavas G, Malliaropoulos N, Pruna R, Traster D, Bikos G, Maffulli N. Neuroplasticity and anterior cruciate ligament injury. Indian J Orthop. 2020;54(3):275-80.

38 Myer GD, Ford KR, Brent JL, Hewett TE. Differential neuromuscular training effects on ACL injury risk factors in "high-risk" versus "low-risk" athletes. BMC Musculoskelet Disord. 2007;8:39.

39 Sugimoto D, Myer GD, McKeon JM, Hewett TE. Evaluation of the effectiveness of neuromuscular training to reduce anterior cruciate ligament injury in female athletes: a critical review of relative risk reduction and numbers-needed-to-treat analyses. $\mathrm{Br} \mathrm{J}$ Sports Med. 2012;46(14):979.

40 Cavanaugh JT, Powers M. ACL rehabilitation progression: where are we now? Curr Rev Musculoskelet Med. 2017;10(3):289-96. 